\title{
Importance of purine metabolites in preeclampsia and acute cerebral stroke
}

Oreshnikov E., Oreshnikova S., Oreshnikov A.

Chuvash State University - Cheboksary (Russia)

\section{Background and Goal of Study: Along} with the classic triad edema, proteinuria, hypertension, more than a quarter century, many clinicians as an indicator of preeclampsia using the high content of uric acid in blood serum hyperuricemia.

It was also found that the hypoxanthine, xanthine and uric acid (UA) are present in the brain, and their content is changed after ischemia, UA is the end product of purine degradation in the brain, xanthine oxidase is also present in the brain, it catalyzes the oxidation of hypoxanthine to xanthine, and then in UA and can be a source of free radicals, inhibition of xanthine oxidase and exogenous administration of UA accompanied by explicit antiischemic and neuroprotective effects in the experiment and clinic, while the endogenous increased its production, with the "side" synthesis of xanthine oxidase oxygen free radicals, reflects the severity of ischemic and reperfusion injury.

We also know that most fatal path pathogenesis (and tanatogenesis) in preeclampsia the development of cerebral stroke.

Our attention was attracted by a comparative assessment of the features of purine metabolism in women with preeclampsia and acute cerebral stroke
Materials and Methods: The study involved 33 patients with preeclampsia and 350 patients in the acute period of cerebral stroke, in which, in addition to conventional laboratory parameters were determined in the blood and cerebrospinal fluid of guanine, hypoxanthine, adenine, xanthine and uric acid direct spectrophotometry

Results and Discussion: It was established that between preeclampsia and cerebral stroke, there are clinical and pathobiochemical parallels, including according to the characteristics of purine metabolism. Hyperuricemia the most famous and at the same time the most pronounced adverse metabolic factor (marker, predictor) for preeclampsia, and for cerebral stroke. High value content oxypurines (hypoxanthine, xanthine and uric acid) in the cerebrospinal fluid a good sign for a stroke, and low for preeclampsia.

Conclusion(s): Liquor can be seen not only as a medium of administration of drugs for spinal anesthesia, but also and a source of valuable diagnostic (and predictive) information, including in preeclampsia. The level of uric acid and other purine both patients with preeclampsia, and cerebral stroke, it is desirable to investigate not only in serum but when possible, and in cerebrospinal fluid. 